\title{
On the Possibility of Developing Matched Acousto-Optical Light Filtering Method for Incoherent Telecommunications and Earth Remote Investigations
}

\author{
V.V. Proklov*, O.A. Byshevski-Konopko And A.V. Lugovskoi \\ Fryazino Branch, Kotel'nikov Institute of Radio Engineering and Electronics, Russian Academy of Sciences, \\ 141190 Fryazino, Russia
}

\begin{abstract}
This is a systematic study of the possibility of creating multi-frequency matched acousto-optic light filters and constructing on new basic principles of the non-coherent optical transmission systems with optical code division multiple accesses and hyperspectral sensors for remote sensing using pre-detector optimal filtering of optical information.
\end{abstract}

DOI: $10.12693 /$ APhysPolA.127.29

PACS: 42.79.Jq, 43.58.Kr, 42.30.-d, 42.79.Sz, 42.79.Qx, 84.40.Ua

\section{Introduction}

Modern acousto-optics (AO) is a highly advanced scientific and technical direction, having a wide range of practical applications. The physical basis for the majority of AO applications is the effect of synchronous interaction of sound and light waves in their joint propagation in photoelastic medium, which results in the diffraction of the incident light [1-4]. Efficiency of AO diffraction in this case may be quite high (up to $\approx 100 \%$ ), the angles of the exiting light beam deflection from the initial beam propagation direction, related with the sound frequency, may vary over a rather wide range, for example, the number of the output beam positions distinguishable by the Rayleigh criterion, may reach $N \approx 500$, with the possibility of their rapid $(\approx 1 \mathrm{~ms})$ and an arbitrary switching. Currently, many of these properties are widely used in order to control the light beam parameters - intensity modulation, polarization changes, deviations and carrier frequency shift, as well as for functional processing of the input optical (acoustic) information such as spectral and correlation analysis of microwave signals with a large "base" $\left(B T>10^{2}\right.$, where $B$ and $T$ - the frequency and duration of the signal), the optical tunable band-pass filtering in spectroscopy systems and optical emission spectroscopy, monitoring the composition of the liquid, the environment, etc.

However, reviews of the literature in the field of acousto-optics show that up to the present such areas as the interaction of incoherent polychromatic light with a multi-frequency sound, the possibility of providing the specified combinations of the spectral composition of light and sound with the suppression of intermodulation distortions, realization of linear algebra operations

* corresponding author; e-mail: proklov@inbox.ru and other transformation of information in the spectral range, etc. are still little-investigated and not well linked to each other.

The deeper study of these areas may allow significantly expand the range of $\mathrm{AO}$ applications, for example, to carry out the evolution of the modern methods of the tunable $\mathrm{AO}$ filtering to more complex functional types of processing of optical signals and images, such as spectral correction of signals, increasing the contrast of images, or even a coherent reception of signals against the interference background.

This paper presents the results of new research in these fields and discusses examples of potentially promising areas of application, such as non-coherent optical communication systems with optical code division multiple access (O-CDMA) and hyperspectral sensors for AO systems for remote sensing of the Earth.

\section{The fundamental physical researches in the field of filtering of optical signals and images}

The first publications on the operating principles and some of the major schemes for constructing tunable $\mathrm{AO}$ filters of optical radiation, in which the spectral bandpass filtering of light was carried out by a sequential (sometimes arbitrary) time adjustment of the sound central frequency [5,6], appeared more than 40 years ago. The operation of such devices is based on the main condition for high performance AO diffraction - the Bragg matching for wavelengths of light and sound, from which it follows that the angle of light deflection is related to the wavelength of light $\lambda$ and the sound frequency $f_{\mathrm{S}}$ by the relation $\theta=F\left(\lambda f_{\mathrm{S}}\right)$, which implies that for a fixed angle of diffraction $\lambda f_{\mathrm{S}}=$ const.

In the early 1990s some ideas appeared about how to use the $\mathrm{AO}$ interaction in planar and strip waveguide structures, which provides coupling between the multifrequency sound and multi-wavelength light, making it possible to perform operations of scalar multiplication 
of the spectrally encoded optical and acoustic signals, on the base of which analog and digital vector-matrix multipliers were developed [7-9].

Somewhat later, in a number of papers the principle of the correlation reception of optical signals with unipolar code modulation of their spectra, based on a "balanced scheme" using weighted "direct" and "complementary" binary codes, has been proposed [10-12]. The main advantage of using the balanced reception scheme is the ease of implementation of the incoherent method for receiving (transmitting) optical unipolar coded signals to overcome their "imbalance", complicating the amplitude-phase realization of the optimal correlation reception usual for conventional radio frequency telecommunication systems.

The question of the active use of a multi-band (with multi-frequency sound) AO filtration requiring high diffraction efficiency has not been considered for a long time since in classic work by Hecht [13] it was shown that in the course of the AO interaction of light with multifrequency sound in the general case some special inherent mechanisms of nonlinearities, caused by intermodulation effects of higher orders, arise.

However, in recent years, in a number of papers [14-16] there were established conditions for significant suppression of signal's spurious intermodulation effects by means of the properly selected geometry of the anisotropic AO diffraction in the crystalline medium and the corresponding synthesis of controlling acoustic signals.

Finally, the first proposals appeared recently on the use of multi-frequency AO diffraction for a multi-pass band filtering of optical images, capable to perform a certain types of adaptive spectral processing (an image contrast enhancement, the correction of the short optical pulses form, etc.) [17-21].

From the above it follows that a notable evolution in the methods of AO light filtering from a relatively simple procedure of slowly time-tunable narrowband to adaptive broadband, which is able to correct the spectral composition of the transmitted light, has already taken place. It should be noted, however, that in this way the problem of AO matched (correlated) filtering of signals from noise interferences with the use of their a priori known characteristics has not been solved yet. This will be discussed in the next section.

\section{Promising methods of AO matched filtering and possible fields of their implementation \\ 3.1. Optical telecommunication system with $O-C D M A$}

\subsubsection{Formulation of the problem}

The problem of constructing low-cost and sufficiently high capacity optical transmission systems based on the principle of O-CDMA involves solving the specific problems associated with the need to use unipolar codes (due to the incoherence of the light used), as well as finding the reliable sources of sufficiently uniform broadband optical radiation and controlled optical dispersion devices for spectral encoding and decoding of light over a sufficiently wide range of wavelengths. Currently, the most promising units for this purpose, from our point of view, are: optical diodes (such as LEDs) as a broadband incoherent light sources and multi-frequency AO filters as tunable dispersion elements and devices for correlation processing of encoded signals.

In particular, for constructing incoherent optical transmission systems, the initial theoretical and experimental study of operation principles of AO elements realizing the digital information spectral encoding have been carried out. For example, in [11] the first experimental attempt of spectral coding of incoherent light by 15-bit $m$-sequence (at wavelengths $\lambda=850-950 \mathrm{~nm}$ with a spectral resolution $\delta \lambda=0.4 \mathrm{~nm}$ ) on the basis of the AO interaction of surface acoustic waves and optical modes in planar Ti-diffused waveguides in lithium niobate $\left(\mathrm{LiNbO}_{3}\right)$ was made. Somewhat later [12], on the basis of the similar waveguide technology there have been proposed concepts of the fiber optic data transmission based on the principle of O-CDMA and encouraging assessments of their main technical and information parameters were given. In general, all the previous works in this area revealed general ideas about the construction principles and possible parameters of optical lines using AO matched filtering (AOMF), since under the development of information systems based on analog devices it is very important to take into account the quality and accuracy of functional transformations during signal processing, which requires further more detailed analysis of this technology properties*.

\subsubsection{New approach}

In connection with the above, in this paper the consideration of a particular concept of building the information channel in the fiber optic O-CDMA system, which uses two identical multi-frequency AO filter (MAOF) both as at the incoherent light coding stage, and at its decoding on the communication lines output (Fig. 1a), is carried out.

The circuit includes a common pulse generator, which controls clocking, periodicity and duration of the bits $(\approx 1 \mathrm{~ns})$, transmitted simultaneously by all users, the number of which is equal to $M$. Each of $M$ users has his own MAOF and an electrically modulated broadband incoherent light source such as luminescent light-emitting diode (LED). All MAOFs and LEDs should be identical. Spectra of control signals of each AO filter are strictly individual and correspond to a coding function, allocated to them. Each AO encoder (Fig. 1b) consists of an electro-optic commutator (EOC), which directs to the encoder output either a signal from the MAOF-1/0 output, or from MAOF-1/1output, depending on the value of the information bit ("0" or " 1 "). Next, formed in this way in-

* In particular, as noted in [22], at the analysis of potential noise immunity of data transmission systems, utilizing analog processors with their inherent "imperfections", it is essential to take into account the relevant "information losses". 


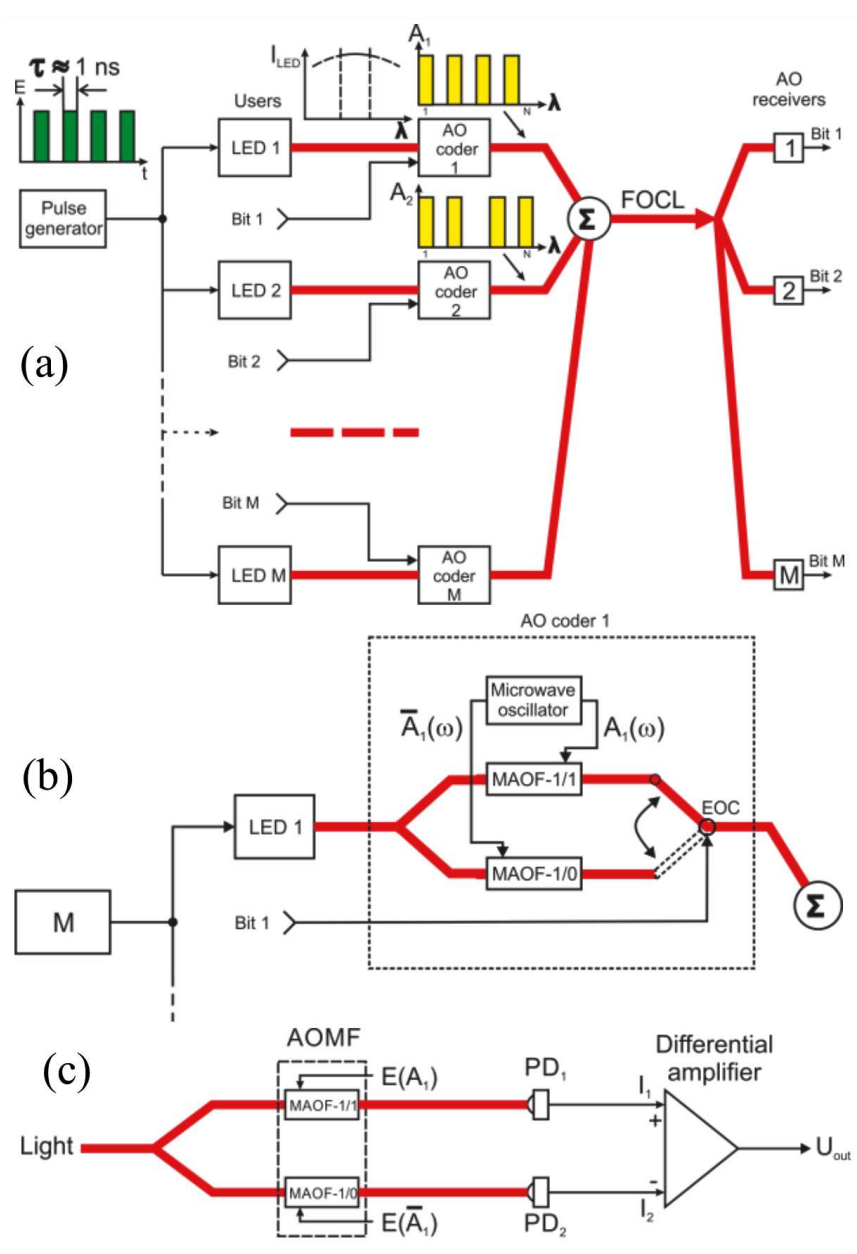

Fig. 1. Schematic diagrams of O-CDMA fibre-optic communication line with acousto-optical coding and decoding (a), AO coder (b), balanced $\mathrm{AO}$ receiver for user 1 (c).

dividual "spectrally distinguishable optical bits" from all users are sending into a common fiber optical communication line. When the switching time of the EOC is more than $\approx 1$ ns then the channel bandwidth is limited by EOC performance, rather than the MAOFs parameters. At that, reprogramming times of the codes are determined by the switching operating response of $\mathrm{AO}$ filter $(\approx 10 \mu$ s), i.e. this can be performed quite quickly.

At the output of the transmission line the entire optical data stream splits into $M$ equal parts (by number of users), in each of which the individual matched processing of aggregate signal is carried out for extraction and identification of "own" bit against of the "alien" ones (Fig. 1c).

It is important to note that in incoherent O-CDMA systems, unlike coherent CDMA radio systems, code functions contain only positive components. It limits application of the regular correlation reception method using direct and their conjugated codes. However, as previously noted, in this case the "balanced reception" method helps out. Thus, the light coming to the user 1 is divided into two equal parts, each of which undergoes parallel filtering by identical $\mathrm{AO}$ filters with different spectral functions of control signals - in the first case, the function is identical to the direct and in the second - to the complementary code of the user.

With a proper selection of the code family the photoelectric detector output in the first channel will include the autocorrelation signal from a given user and the sum of all cross-correlation responses from other users. The second channel output contains all of the same cross-correlation responses without the autocorrelation signal, and hence the difference between them will give the desired discrimination the "own" bit from the "aliens" background.

The choice of coding sequences is the matter of a great importance. Till now many comparable alternatives, like $m$-sequences, Hadamard and Walsh codes, and etc. have been considered [10-12, 23]. Here we use the Walsh 8bit codes as it was done in the earlier proposed scheme O-CDMA based on MAOFs [23].

We will consider further the modeling of AOMF operation on the receiving side (see Fig. 1c). The light signal is directing to the MAOF-1/1 on which acoustic input the control signal, corresponding to direct chosen Walsh function, is entering. In the second channel (MAOF$1 / 0$ ) the control signal corresponds to the complementary Walsh function. The output light signals of both MAOFs are the results of a matched spectral filtering. Next, signals from the MAOF- $1 / 1$ and MAOF- $1 / 0$ outputs are integrated on the respective photodetectors $\left(\mathrm{PD}_{1}\right.$ and $\left.\mathrm{PD}_{2}\right)$ and fed to the differential amplifier. The signal from the amplifier output $U_{\text {out }}$ is analyzed for making a decision on the presence or absence of the bit corresponding to the current user as well as on the sign of this bit in the case of its presence.

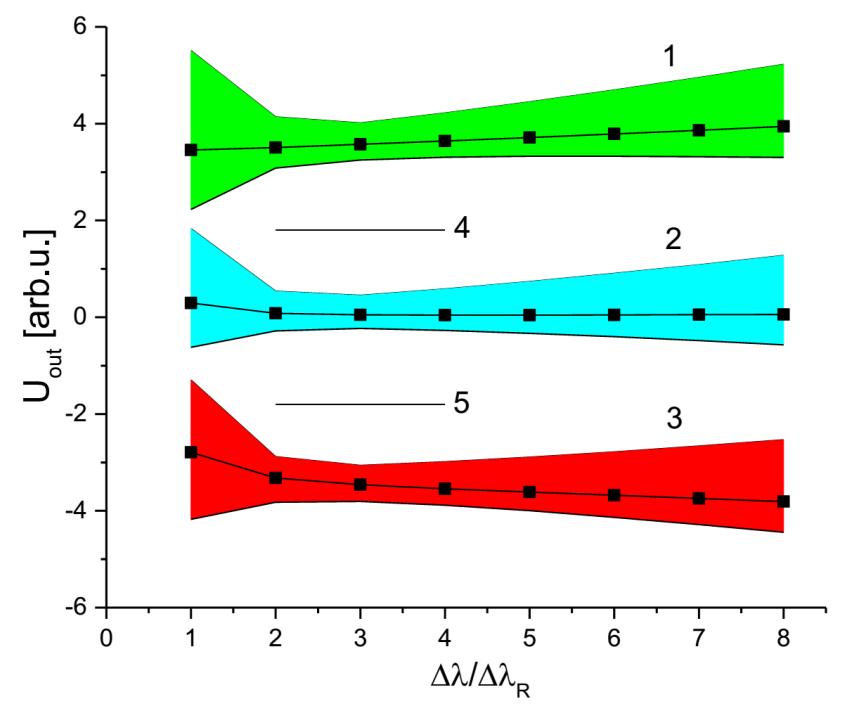

Fig. 2. Modelling of optimal spectral separation.

This paper presents the simulation of data throughput of the proposed system, using a 16-bit Walsh codes at data transmission in a line with 12 active users. 
The $U_{\text {out }}$ value was calculated for all possible combinations of transmitted signals, taking into account all variants of the presence or absence of signals in any of the 12 channels and all possible signs of transmitted bits in conjunction with going through all the numbers of the receiving channels. Analysis of the simulation results is shown in Fig. 2.

It need to be underlined that for the primary estimation of the telecommunication link capability one has to take into account possible values of the signal to interference ratio (autocorrelation to cross-correlations) with a negligible influence of noise.

The abscissa axis values represent the separation of neighboring spectral lines $\Delta \lambda$, which is expressed in the intervals of wavelengths $\Delta \lambda_{\mathrm{R}}$, corresponding to the Rayleigh resolution criterion between adjacent grid points $\lambda_{i}$. The areas with numbers 1,2 and 3 correspond to all possible cases, when any user either sends " 1 " or does not transmit anything or transmits "0", respectively. All possible $U_{\text {out }}$ values lie in Fig. 2 shaded areas. Lines with square markers within these areas show the average $U_{\text {out }}$ value for all variants of interfering signals from other users.

It is clearly seen that between shaded areas, there are zones in which $U_{\text {out }}$ never gets. This makes it possible to select the threshold levels for the triggering of the decision-making device. For example, if $U_{\text {out }}$ lies above the level of line 4, the decision to accept "one" is making. If $U_{\text {out }}$ lies below the level of line 5 , a decision is to accept "zero". The $U_{\text {out }}$ value, lying between the lines 4 and 5 , means that the signal was not transmitted at all. Having analyzed the dependence of the vertical size of the two gaps (the first one - between areas 1 and 2, and the second - between areas 2 and 3 ) on the interval between the spectral components $\lambda_{i}$, one can conclude that $\Delta \lambda / \Delta \lambda_{\mathrm{R}}$ value equal to two- or threefold Rayleigh criterion is optimal from the point of view of the correct decision probability increasing at the minimum width of the used spectral range.

It should be noted that the calculation accuracy of the auto- and cross-correlation functions for spectral binary code sequences, naturally, requires the implementation of a sufficiently high identity of their significant spectral components amplitudes, which is a particular problem at the stage of signal synthesis at the encoder, since the required spectral signal range is usually comparable to the LED envelope energy spectrum width.

For this reason the method of the "one's" code components "equalization" on the example of the AO generator based on the use of the non-collinear geometry $\mathrm{AO}$ interaction in $\mathrm{TeO}_{2}$ and commercially available light-emitting diode in the near infrared wavelength range (900 to $980 \mathrm{~nm}$ ) has been studied. Figure 3 shows results of LED continuous power spectrum measurements (dotted line) and its "equalization", representing the worst case from five 8-bit Walsh functions, used at the measurements.

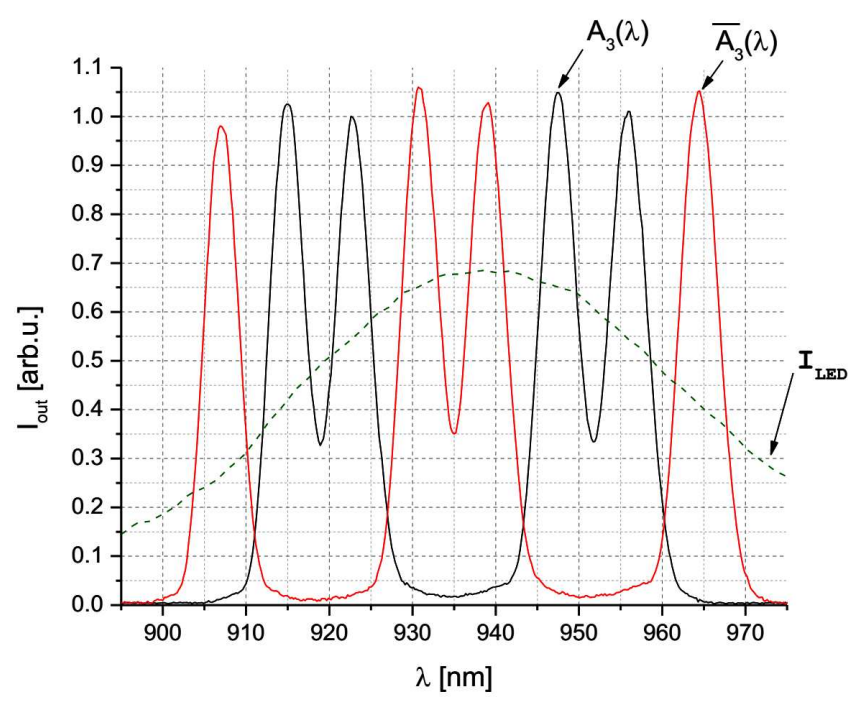

Fig. 3. Experimental verification of bit equalization in AO-coder for one user, showing the worst case from five 8-bit Walsh functions.

The effect of the code "one's" equalization in the entire signal band was achieved by an appropriate optimal synthesis of multi-frequency signal with suppressed intermodulations [14-16]. Achieved uniformity of code "one's" (chips) $\approx 5 \%$ for this system is quite acceptable, because, as shown by the calculations, during the decoding process it does not decrease the expected maximum data transfer rate for the considered system, built on a modern elementary base, which in the optical range may reach a value up to $5 \times 10^{9} \mathrm{bits} / \mathrm{s}$.

\subsection{Acousto-optic matched signal processing in hyperspectral Earth remote sensing}

\subsubsection{Problems of signal processing in modern optical} Earth remote sensing

In the past decade Earth remote sensing (ERS) optical imaging systems with a large number of spectral channels, that are been installing on aircraft and space platforms [24], are more and more widely used. Hyperspectral ERS system, aimed at the significant expanding the range of tasks in the field of remote sensing, should cover a wide spectral range, including visible and infrared ones, with a spectral resolution from a few to tens of nanometers. In this case real-time data processing, received on the aircraft board, often becomes difficult to fulfill.

Indeed, the volume of "data cube", actively used under hyperspectral Earth remote investigations [24], containing the spatial and spectral characteristics of ground objects, constantly accumulated in the recently explored system during only one "frame" recording, can reach gigabytes during a few seconds. Obviously, this leads to serious problems not only for information processing on board, but and with its accumulation and transfer to the ground processing centers.

Many of the problems are caused mainly by currently used approach, in which the input stream of optical information (the original color image area) is divided into 
a set of $N$ narrowband channels ending with the photodetecting matrixes $\left(P M_{i}\right)$ which record the distribution of the light intensity in the image at selected wavelengths $\lambda_{i}(i=1,2, \ldots N)$. Thus, a large number of "narrow-band" analog images, which are processing further in high-performance computers, are obtained at the output of $N$ channels.

In this regard, the principles of acousto-optic adaptive and matched filtering of optical signals, discussed in this paper, seem to be very actual, since they are able in principle to perform "pre-detector optical information processing".

3.2.2. About possibility of using MAOF in promising hyperspectral optical sensors

As already noted, the spectrum of the light transmitted through MAOF with a given spectral transmission function corresponds with the product of the incident light spectral function to the transmission spectral function of AO filter. In particular, in Sect. 3.1, devoted to O-CDMA, signal processing of coded spectral sequences, belonging to the same orthogonal Walsh family, is based on this correlation. However, as it will be shown in the forthcoming discussion in this section, this approach may be applicable also to the procedure of unmixing the reflectance spectra of light from different objects of nature.

\subsubsection{To illustrate, consider the following problem}

Let the "white" incoherent light with an intensity $W_{0}=\operatorname{const}(\lambda)$, where $\lambda$ is the wavelength, illuminates uniformly a ground pixel with area $\Phi_{0}$. Relative part of this area $k_{1}$ is occupied by one of the possible types of objects, belonging to a finite set $A_{j}, j=1,2,3 \ldots M$ (a subpixel target), while the remaining relative part of the pixel $k_{2}$ - by a known type of background $B_{0}$, typical for an investigated terrain $\left(k_{1}+k_{2}\right.$ must be equal to 1$)$. There is a library [25] of etalon spectral energy reflection coefficients $R^{\text {et }}\left(\lambda_{i}\right)$ tables for all types of backgrounds and objects, where the discrete set of $\lambda_{i}$ belongs to a wide (hyperspectral) wavelength range of light.

By means of fast matched spectral processing of the light, arriving from an each of many ground pixel in line ${ }^{\dagger}$, it is required to determine the most probable type of object (or a part of it, located in this pixel) for a known type of background.

The proposed new method of the problem solution is based on the use of two identical MAOFs, having different spectral transmission characteristics $F(\lambda)$.

Let us note also that the spectral transmission of the AO filter with low diffraction efficiency $\eta(\lambda)_{\max } \ll 1$ has a linear form $[1,2]$ :

\footnotetext{
†We are analyzing one line of 2D image of scene, situated normally to the flying vehicle trajectory; herewith the light from all the elements of the line (ground pixels) is coming and filtering in a common AO receiver simultaneously. After this, results of the matched filtering of all the corresponding signals from each pixel are accumulated on the output photoreceiver detector line.
}

$$
F\left(\lambda-\lambda_{i}\right)=\eta_{\max } C_{0} P_{\mathrm{S}}\left(\lambda_{i}\right) \operatorname{sinc}^{2}\left(\Delta \beta_{i}\right),
$$
where $C_{0}-$ a constant depending on the parameters of the medium and the geometry of $\mathrm{AO}$ interaction, $\Delta \beta_{i}=2 \pi \Delta n L\left(\lambda-\lambda_{i} / \lambda_{i}^{2}\right)$ - the value of the phase mismatch of AO interaction synchronism, $L$ - the length of AO interaction, $P_{\mathrm{S}}\left(\lambda_{i}\right)$ - the sound power at frequency $f_{i}$ at carrying out the spectral filtering of light with wavelength $\lambda_{i}$, taking into account the relation $\lambda_{i}=\Delta n V_{\mathrm{S}} / f_{i}$, where $\Delta n=n_{\mathrm{e}}-n_{\mathrm{o}}$ - optical anisotropy factor and the $V_{\mathrm{S}}$ - the phase velocity of sound in crystal, respectively.

From this it follows that when passing through MAOF with the spectral characteristic $F(\lambda)$ the input spectrum is multiplied scalarwise by the filter spectrum function, i.e. output signal undergoes complex filtering. For example, for the signal from an object $A_{\text {in }}$ we obtain at the filter output

$$
A_{\text {out }}(\lambda)=\left[A_{\text {in }}(\lambda) \bullet F(\lambda)\right] .
$$

That is after passing through the filter all of the light reflected from the background and the object areas, with its subsequent photoelectric conversion and detection, the output power is obtained by the sum of the two filtered signals

$$
I_{\mathrm{MAOF}}=W_{0} \Phi_{0}\left(k_{1}\left[R_{A_{\text {in }}} \bullet F\right]+k_{2}\left[R_{B_{\text {in }}} \bullet F\right]\right) .
$$

To solve this problem it is required, in general, to split all the light into two equal beams and simultaneously perform their AO-filtration using two identical MAOF (see Fig. 4) in which filtration procedures should be matched the normalized spectra of the signals, corresponding to the presupposed type of the object $A_{\text {in }}$ and the specified type of background $B_{0}$ :

$$
\begin{gathered}
\text { MAOF-1 : } F_{N}^{A}(\lambda)=\sum_{i=1}^{N_{\mathrm{SEL}}}\left(R_{A \text { in }}^{\mathrm{et}}\left(\lambda_{i}\right) / R_{A \max }^{\mathrm{et}}\right) \bullet F\left(\lambda-\lambda_{i}\right), \\
\text { MAOF-2 : } F_{N}^{B}(\lambda)=\sum_{i=1}^{N_{\mathrm{SEL}}}\left(R_{B_{0}}^{\mathrm{et}}\left(\lambda_{i}\right) / R_{B \max }^{\mathrm{et}}\right) \bullet F\left(\lambda-\lambda_{i}\right),(4)
\end{gathered}
$$

where $N_{\mathrm{SEL}}$ - the number of spectral lines, selected from etalon reflection coefficients tables and $R_{A_{\max }, B_{\max }}^{\text {et }}$ - maximum values of reflection coefficients among selected spectral lines.

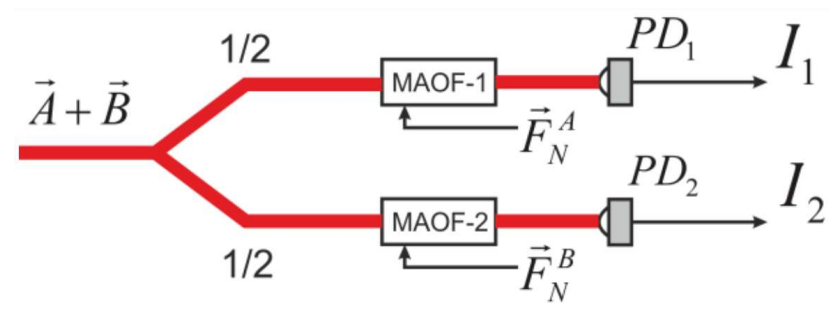

Fig. 4. Schematic diagram of receiver on the base of MAOF.

Note that in the hyperspectral processing literature the representation of spectral signals in multi-dimensional vector space [24] is widely used, therefore the MAOF action can be interpreted as scalar product of two vectors lying in the same plane. This allows to determine at the 
filters output the sum of power projection of the individual signals since incoherent light spectral components are uncorrelated. As a result, for the solution of the problem one can create and solve a system of equations for the AO experimental computational method for determining the power of the incoming signals.

In this case, it is simplest to carry out measurements using MAOF-1 and MAOF-2 filters (channels 1 and 2 in Fig. 4), which adapted respectively to the optimal filtering of any of the possible hypotheses $A_{\text {in }}$ and to the background signal $B_{0}$. From these measurements we can immediately determine the most probable type of the identified object $A_{j}(j=1,2, \ldots N)$.

Indeed, according to Eq. (3) one can obtain after filtering

$$
\begin{aligned}
& I_{1}^{*}=k_{1} S_{11}+k_{2} S_{21}, \\
& I_{2}^{*}=k_{1} S_{12}+k_{2} S_{22},
\end{aligned}
$$

where $I_{1,2}^{*}=I_{1,2} /\left(W_{0} \Phi_{0} \eta_{\max }\right)$,

$$
\begin{aligned}
S_{11} & =\int R_{A_{\text {in }}}(\lambda) F_{N}^{A}(\lambda) \mathrm{d} \lambda, \\
S_{21} & =\int R_{B_{0}}(\lambda) F_{N}^{A}(\lambda) \mathrm{d} \lambda, \\
S_{12} & =\int R_{A_{\text {in }}}(\lambda) F_{N}^{B}(\lambda) \mathrm{d} \lambda \text { and } \\
S_{22} & =\int R_{B_{0}}(\lambda) F_{N}^{B}(\lambda) \mathrm{d} \lambda .
\end{aligned}
$$

The solution of Eqs. (5) and (6) for all $A_{j}(j=1,2 \ldots N)$ and a chosen background $B_{0}$ allows to determine the number $j$, corresponding to the most probable hypothesis about the type of object providing that one single pixel contains only two types of objects - the object itself and the background with their respective relative areas $k_{1}$ and $k_{2}$, where $k_{1}+k_{2}=1$. The last condition can be satisfied with only a single correct hypothesis $A_{j}$, while for all other ("wrong" hypotheses for $i \neq j$ ) $k_{1}+k_{2} \neq 1$. This follows from the fact that in these cases the previously mentioned vector projecting occurs under conditions where the incident light vector lies outside the "hypothetical plane".

In order to verify the most essential points of this approach a computer modeling of some the most important stages of AO method for spectral recognition of the signal type for some natural background and object types have been conducted.

For a preliminary estimation of the accuracy characteristics of the proposed $\mathrm{AO}$ recognition method a numerical experiment was carried out in which the object of the study were three natural items with known spectral characteristics: grass (background $B_{0}$ ), and two types of hematite $\left(A_{1}\right.$ and $\left.A_{2}\right)$ [25]. The MAOF parameters based on $\mathrm{TeO}_{2}$ described in Sect. 3.1 of this article (see also Fig. 3) were used. In the hyperspectral range $(0.5-2.5 \mu \mathrm{m}) 10$ spectral lines with the most expressed differences in the reflection coefficients were selected for each of the three objects. These selected values were then "transferred" to the working range of available
MAOF as the corresponding grid of 10 spectral lines, placed at intervals equal to 3 times spectral resolution by the Rayleigh criterion for the AO filter.

The calculations with use of Eqs. (5) and (6) show that with the available accuracy of AO measurements and a reasonable high identity of MAOF-1 and MAOF-2 $\left(\delta I_{1,2} / I_{1,2} \approx 1 \%\right)$ the discrepancies of $\operatorname{sum}\left(k_{1}+k_{2}\right)$ from 1.0 for the "right" and "wrong" hypotheses $\left(\Delta_{\mathrm{R}}\right.$ and $\left.\Delta_{\mathrm{W}}\right)$ form two rather well distinguishable areas

$$
\Delta_{R} \approx 0.0 \pm 0.01 \text { and } \Delta_{W} \approx 0.06 \pm 0.01
$$

with the sufficiently wide "empty" interval $\approx 4 \%$ between them, which makes it possible to use a threshold method of differentiation the correct hypothesis from incorrect ones.

\section{Conclusion}

1. The first systematic consideration of the O-CDMA system parameters with application of the spectraldomain signal coding/decoding by the family of Walsh sequences is presented. Transmission link characteristics were estimated via computer simulation using the noncollinear geometry of $\mathrm{AO}$ interaction in $\mathrm{TeO}_{2}$ crystal over IR wavelengths range.

The modeling results show that the system can operate with the frequency separation between adjacent spectral lines of at least double/triple the Rayleigh criterion. In this case, the signal-to-noise ratio provides a threshold for making the decision concerning the received bit value.

It is also shown that at an optimal choice of the acoustic signal spectra, the considered asynchronous data transmission system with the selected 16-bit length Walsh family enables to obtain the following potential parameters:

- light wavelength range, $900-980 \mathrm{~nm}$;

- intervals between adjacent spectral lines, $5 \mathrm{~nm}$;

- the number of simultaneously serviced users (channels), 12;

- and the highest speed of data transmission in the operation mode, up to $5 \mathrm{Gbit} / \mathrm{s}$.

2. For the first time, application of the MAOFs as the efficient tool for a signal processing in the hyperspectral sensors capable of the pre-detector matched filtering of optical information is proposed. Such primary AO processing can substantially accelerate a decision making process in hyperspectral systems and appreciably diminish the data volume for post-processing procedures with use of modern onboard computers.

\section{Acknowledgments}

The authors are grateful to Yu.V. Pisarevskii for very valuable experimental assistance, as well as to A.L. Filatov and A.V. Gerus for fruitful discussions during preparation of this manuscript.

This study was supported by the Russian Foundation for Basic Research, project N 12-07-00186-a and 13-02-00479-a. 


\section{References}

[1] Yu.V. Gulyaev, V.V. Proklov, G.N. Shkerdin, Sov. Phys.-Usp. 21, 29 (1978).

[2] V.I. Balakshii, V.N. Parygin, L.E. Chirkov, Basic Physics of Acoustooptics, Radio i Svyaz, Moscow 1985 (in Russian).

[3] Acoustooptical Processors of Spectral Type, Eds. V.V. Proklov, V.N. Ushakov, Radiotekhnika, Moscow 2011 (in Russian).

[4] L.N. Magdich, V.Ya. Molchanov, Acousto-optic Devices and Their Applications, Gordon and Breach Sci., New York 1989.

[5] I.C. Chang, Appl. Phys. Lett. 25, 323 (1974).

[6] I.C. Chang, Opt. Eng. 16, 455 (1977).

[7] V.V. Proklov, A.U. Bashlakov, Yu.V. Gulyaev, S.V. Sokolovsky, Int. J. Opt. Comput. 1, 141 (1990).

[8] N. Goto, Y. Miyazaki, Trans. IEICE E70, 981 (1987).

[9] V.V. Proklov, O.A. Byshevski-Konopko, V.N. Kurskii, in: Proc. 1999 IEEE Ultrasonic Symp., Eds. S.C. Schneider, M. Levy, B.R. McAvoy, IEEE, New York 1999, p. 17.

[10] M. Kavehrad, D. Zaccarin, J. Lightwave Technol. 13, 534 (1995).

[11] I. Hinkov, V. Hinkov, K. Iversen, O. Ziemann, Electron. Lett. 31, 384 (1995).

[12] V.V. Proklov, in: Proc. 8-th Spring School "AcoustoOptics and Applications" and 6-th EOS Meeting "Advances in Acousto-Optics" AA-O.01, Eds. A. Sliwiński, P. Kwiek, B. Linde, A. Markiewicz, University of Gdanśk, Gdańsk 2001, Poland, p. 11.
[13] D.L. Hecht, IEEE Trans. Son. Ultrason. 24, 7 (1977).

[14] V.V. Proklov, S.N. Antonov, Yu.G. Rezvov, A.V. Vainer, in: Proc. 2006 IEEE Ultrasonics Symp., Ed. M.P. Yuhas, IEEE, New York 2006, p. 248.

[15] S.N. Antonov, A.V. Vainer, V.V. Proklov, Yu.G. Rezvov, Tech. Phys. 53, 752 (2008).

[16] S.N. Antonov, A.V. Vainer, V.V. Proklov, Yu.G. Rezvov, Appl. Opt. 48, 171 (2009).

[17] P.I. Shnitser, I.P. Agurok, Proc. SPIE 3140, 117 (1997).

[18] J. Liu, R. Shu, Y. Ma, J. Wang, Proc. SPIE $\mathbf{7 8 5 7}$ $78571 \mathrm{~K}-1$ (2010).

[19] V.Ya. Molchanov, S.I. Chizhikov, O.Yu. Makarov, N.P. Solodovnikov, V.N. Ginzburg, E.V. Katin, E.A. Khazanov, V.V. Lozhkarev, I.V. Yakovlev, Appl. Opt. 48, C118 (2009).

[20] B.S. Gurevich, S.V. Andreyev, A.V. Belyaev, V.N. Chelak, Proc. SPIE 5828, 53 (2005).

[21] V.Ya. Molchanov, K.B. Yushkov, Opt. Express 22 15668 (2014).

[22] Yu.B. Sindler, V.V. Proklov, V.I. Grigor'evskii, O.A. Byshevskii-Konopko, V.N. Kurskii, J. Commun. Technol. Electron. 53, 1225 (2008).

[23] V.V. Proklov, O.A. Byshevski-Konopko, V.I. Grigorievski, Quant. Electron. 43, 542 (2013).

[24] D. Manolakis, D. Marden, G.A. Shaw, Lincoln Laboratory J. 14, 79 (2003).

[25] USGS Digital Spectral Library 06, http://speclab.cr.usgs.gov/spectral.lib06/. 